\title{
A Partial Delivery Bi-Objective Vehicle Routing Model with Time Windows and Customer Satisfaction Function
}

\author{
Maral Shahmizad ${ }^{*_{a}}$ \\ Saeed Khanchehzarrina \\ Iraj Mahdavia \\ Nezam Mahdavi-Amirib \\ a Department of Industrial Engineering, Mazandaran University of Science and Technology, Babol, Iran \\ ${ }^{b}$ Faculty of Mathematical Sciences, Sharif University of Technology, Tehran, Iran \\ *Corresponding Author Email: Maralshahmizad@yahoo.com
}

\section{Doi:10.5901/mjss.2016.v7n4s2p102}

\section{Abstract}

\begin{abstract}
We present a new bi-objective model for vehicle routing problem with partial delivery and time windows considering customer's satisfaction. Objectives are to maximize the customer satisfaction and minimize the travel time. Hard time windows and the possibility of multiple visits are considered and the customer needs could be more than the vehicle's capacity. A mixed-integer nonlinear programming model is provided which is linearized to obtain the optimal solutions. A number of random instances are generated and solved by the LINGO to assess and validate the effectiveness of the proposed model.
\end{abstract}

Keywords: Partial delivery vehicle routing problem, Time window, Customer satisfaction function.

\section{Introduction}

Vehicle routing problem (VRP) refers to a set of problems where a fleet of vehicles placed in one or more depots are to serve a set of customers each having a certain demand. Dantzing and Ramser [1] first introduced VRP in 1959. The goal was to determine a set of routes for vehicles having limited capacity so that the total cost is minimized and customer demands are satisfied. There are various forms of VRPs, the most famous of which are the capacitated vehicle routing problem (CVRP) and vehicle routing problem with time window (VRPTW). The capacity restriction is that the total demand of customers assigned to the vehicles should not be greater than the vehicles capacity and the time window constraints imply that the service time for customers should be delivered in the time period set by customers $[2,3]$. In the past decades, various studies have been carried on VRPTW and CVRP $[4,5,6,7]$. In today's competitive environment, customer satisfaction plays an important role in the success of service and manufacturing companies. One important aspect that elevates customers' satisfaction in both service and manufacturing systems is the quickness in the delivery of services or products to customers. Research on minimum latency problems (MLPs) has been conducted to achieve this desire from the transportation and logistics viewpoints. MLP is also known as cumulative vehicle routing problem (Chen et al. [8], 2012) or Customer Centric Vehicle Routing Problem (Martinez-Salazar et al. [9], 2014), which is a class of routing problems with the objective to minimize the waiting time of customers. Customer satisfaction and competitive business environment, on the one hand, and increase in the number, scale, and severity of disasters, on the other hand, are key elements for MLP to receive considerable attention from researchers. Different variants of the problem have been developed from 1986 to 2015 (see [10]). In the classical VRP it is assumed that all customer demands are lower than the vehicles' capacity, and thus vehicles are assigned to nodes in such a way that they can respond to all customer demands. However, in practice, due to resource limitations, it is possible that customer demands be greater than a vehicle capacity. In this case, the customer demands cannot be met in just one visit. These kinds of problems are called split delivery vehicle routing problem and were first introduced by Dror and Trudeau [11, 12]. Fig.1 illustrates an example of a simple network with three customers, where the edge labels are distances, the node labels are demands (or delivery amounts), and the vehicle capacity is 100. In its optimal solution (Fig. 1a), there are three routes without split deliveries, and the total distance traveled is 48. The problem is equivalent to a SDVRP; and its optimal solution (Fig. 1b) shows that customer 2 receives two deliveries from two separate routes. In 1994, Dror [13] presented an integer linear programming 
model for SDVRP. In 1995, Giffen and Fizell [14] presented a mixed integer programming model for SDVRP and considered hard time windows. In 2001 and 2005, Archetti et al. [15, 16] introduced K-SDVRP which is a special case of SDVRP with the vehicle capacity $\mathrm{K} \in Z^{+}$. In 2006, Archetti et al. [17] presented a worst cast analysis for SDVRP showing that in split delivery saving is up to $50 \%$. Archetti et al. [18] in 2008 showed that most reduction is achieved when the average customer demand is 50 to 70 percent of the vehicle capacity and variation of demand is low. In recent works, the goal was saving cost and because of that at each stage a part of customer demand may be satisfied. Some exactsolution approaches were developed for determining and improving the bounds of the optimal solution for SDVRP. Belenguer et al. [19] in 2000 performed a polyhedral analysis, explored several valid inequalities, and implemented a cutting-plane algorithm. Jin et al. [20] in 2007 presented a two-stage exact algorithm, while Jin et al. in 2008 improved the bounds generated by Belenguer et al. [19] in 2000 with a column generation algorithm. Recently, Archetti et al. [21] in 2014 presented two branch-and-cut algorithms providing lower bounds for the optimal solution. In 2015, Silva et al. [22, 23] presented a multi-start iterative local search based heuristic named splitlLS [22, 23]. Here, with regards to resource limitations a new version of SDVRP is considered that we call partial delivery vehicle routing problem. In this case, customer demand is satisfied in several stages in a discrete way. This model is used in distribution of essential goods and emergency events with lack of resources.

\section{VRP}
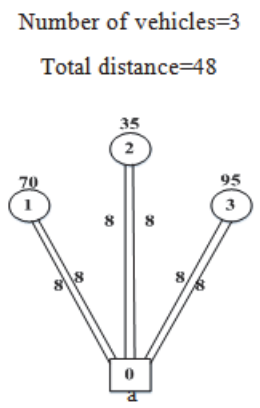

SDVRP

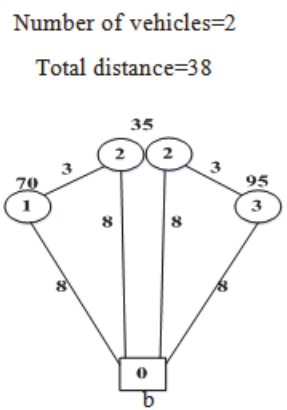

Figure 1. Comparison between VRP and SDVRP

The remainder of our work is organized as follows. The problem is defined in Section 2. A mathematical model is presented in Section 3. Computational results are given in Section 4. Finally, conclusions and future research organized directions are provided in Section 5.

\section{Problem Description}

Here, we present a mixed integer nonlinear programming (MINLP) model for split delivery vehicle routing problem with time window (SDVRPTW). The problem is defined on a complete undirected graph $G=(A, E)$, with $A=\{0,1,2, \ldots, N\}$ as the set of nodes and $E=\{(i, j) \mid i, j \in A, i \neq j\}$ as the set of edges, where $\{1, \ldots, N\}$ denote the set of customers and 0 is considered as the depot. The homogeneous vehicles with the same capacity $Q$ are located at the depot. For arc $(i, j)$, we have $d_{i j}$ and $t_{i j}$ respectively representing the distance and the travel time between the two nodes. Each customer $i$ has:

(1) nonnegative demand $d_{i}$,

(2) time window $\left[a i, b_{i}\right]$, and

(3) service time $s_{i}$.

It is assumed that due to the lack of resources, customer demands are not satisfied in just one visit. The aim is to find a path to minimize the total travel time and maximize the customer satisfaction.

\subsection{Satisfaction function}

A satisfaction function gives the amount of each customer's demand to be met at each period. Demand satisfaction at early stages results in a greater customer satisfaction. Another factor that affects satisfaction of the customer is the number of periods needed to satisfy the customer demands. There is an inverse relationship between satisfaction and the 
number of periods of servicing the customer. To evaluate the satisfaction function, customers are prioritized according to an order to meet the demands of the targeted customers. The relation below shows the impact of two factors on satisfaction function:

$$
C s_{j} \propto \frac{d_{j \ell}}{\ell} .
$$

\subsection{Maximum number of periods of service}

According to the vehicle capacity $(Q)$ and the number of vehicles $(C)$, the maximum number of periods of service is defined by

$$
L=\left[\frac{\sum_{j} D_{j}}{C Q}\right]+1,
$$

where ${ }^{D_{j}}$ is the jth customer demand.

\section{Mathematical Formulation}

Here, we present the following mathematical model for partial delivery vehicle routing problem

\begin{tabular}{|c|c|}
\hline $\begin{array}{l}\text { Inputs: } \\
\{1, \ldots, N\}\end{array}$ & set of indices for customers \\
\hline$\{1, \ldots, K\}$ & set of indices for vehicles \\
\hline$\{1, \ldots, L\}$ & set of indices for periods \\
\hline$D_{j}$ & travel total demand of customer $j$ \\
\hline$t_{i j}$ & time between nodes $i$ and $j$ \\
\hline$a_{j}$ & earliest time to start the service at customer $j$ \\
\hline$b_{j}$ & latest time to start the service at customer $j$ \\
\hline$Q$ & capacity of each vehicle \\
\hline$s_{j}$ & service time for customer $j$ \\
\hline$w_{j}$ & weight of customer $j$ \\
\hline $\begin{array}{l}M \\
\text { Variables: }\end{array}$ & a large positive number. \\
\hline $\begin{array}{l}d_{j \ell} \\
e_{i}\end{array}$ & $\begin{array}{l}\text { an amount demand of customer } j \text { to be satisfied for customer } j \text { in period } I \\
\text { arrival time at node } j \text { in period / }\end{array}$ \\
\hline$u_{i k l}$ & accumulated demand serviced along a route after visiting customer $i$ by vehicle $k$ in period $I$ \\
\hline$C s_{j}$ & the amount of jth customer satisfaction \\
\hline$y_{i k \ell}$ & $\begin{array}{l}1 \text {, if node } i \text { uses vehicle } k \text { in period } l \text {; } \\
0, \text { otherwise }\end{array}$ \\
\hline$x_{i j k \ell}$ & $\begin{array}{l}\text { 1, if in period / vehicle } k \text { goes from node } i \text { to } j \text {; } \\
0, \text { otherwise }\end{array}$ \\
\hline
\end{tabular}

\section{Sets and parameters:}

\subsection{Mathematical model}

$$
\begin{array}{ll}
\max \mathrm{z}_{1} & =\sum_{j=1}^{N} C s_{j}=\sum_{j=1}^{N} w_{j} \sum_{\ell=1}^{L} \frac{d_{j \ell}}{\ell} \\
\min \mathrm{z}_{2}=\sum_{i=1}^{N} \sum_{j=1, j \neq i}^{N} \sum_{k=1}^{K} \sum_{l=1}^{L} t_{i j} x_{i j k l} & \\
\text { s.t. } & \forall j=1, \ldots, N, \forall \ell \\
\sum_{i=0, i \neq j}^{N} \sum_{k=1}^{K} x_{i j k \ell} \leq 1, &
\end{array}
$$




$$
\begin{aligned}
& \sum_{i=0, i \neq j}^{N} \sum_{k=1}^{K} x_{i j k \ell}=\sum_{i=0, i \neq j}^{N} \sum_{k=1}^{K} x_{i j k \ell} \\
& \forall j=0,1, \ldots, N, \forall \ell \\
& \sum_{k=1}^{K} x_{i j k \ell} \leq 1 \\
& \forall i, \forall j=1, \ldots, N, j \neq i, \forall \ell \\
& \sum_{i=0, i \neq j}^{N} x_{i j k \ell}=\sum_{i=0, i \neq j}^{N} x_{j i k \ell}, \\
& \forall j=1, \ldots, N, \forall \ell, \forall k \\
& \sum_{\ell=1}^{L} d_{j \ell} \geq D_{j} \\
& \forall j=1, \ldots, N, \forall \ell \\
& d_{j \ell} \leq D_{j} \cdot\left(\sum_{i=0, i \neq j}^{N} \sum_{k=1}^{K} x_{i j k \ell}\right), \\
& \forall j=1, \ldots, N, \forall \ell \\
& \sum_{j=0, j \neq i}^{N} x_{i j k \ell}=y_{i k \ell} \text {, } \\
& \forall i=1, \ldots, N, \forall \ell, k \\
& -M\left(1-\sum_{i=0, i \neq j}^{N} \sum_{k=1}^{K} x_{i j k \ell}\right)+a_{j} \leq e_{j \ell} \leq b_{j}+M\left(1-\sum_{i=0, i \neq j}^{N} \sum_{k=1}^{K} x_{i j k \ell}\right), \\
& \forall j=1, \ldots, N, \forall \ell, k \\
& d_{j \ell} \leq u_{j k \ell} \leq Q+M\left(1-\sum_{i=0, i \neq j}^{N} x_{i j k \ell}\right), \\
& \forall j=1, \ldots, N, \forall \ell, k \\
& u_{i k \ell}-u_{j k \ell}+Q x_{i j k \ell} \leq Q-d_{j \ell}+M\left(1-\sum_{i^{\prime}=0, i^{\prime} \neq j}^{N} x_{i j k \ell}\right)+M\left(1-\sum_{i^{\prime}=0, i^{\prime} \neq i}^{N} x_{i^{\prime \prime} k \ell}\right), \quad \forall i, j=1, \ldots, N, j \neq i, \forall \ell \\
& \sum_{i=0, i \neq j}^{N} \sum_{k=1}^{K} x_{i j k \ell} \leq d_{j \ell} \\
& \forall j=1, \ldots, N, \forall \ell \\
& d_{j \ell} \leq D_{j}-\sum_{\ell^{\prime}=2}^{\ell} d_{j, \ell^{\prime}-1}, \\
& \forall j=1, \ldots, N, \forall \ell \\
& \sum_{i=1}^{N} x_{0 i k \ell} \leq 1 \\
& \forall k, \forall \ell \\
& e_{i \ell}-e_{j \ell} \leq M\left(1-\sum_{k=1}^{K} x_{i j k \ell}\right)-\sum_{k=1}^{K}\left(t_{i j}+s_{i}\right) x_{i j k \ell}, \\
& \forall i, j=1, \ldots, N, j \neq i, \forall \ell \\
& e_{i \ell}-e_{j \ell} \geq-M\left(1-\sum_{k=1}^{K} x_{i j k \ell}\right)-\sum_{k=1}^{K}\left(t_{i j}+s_{i}\right) x_{i j k \ell}, \\
& \forall i, j=1, \ldots, N, j \neq i, \forall \ell \\
& e_{j 1} \geq t_{0 j} \cdot \sum_{k=1}^{K} x_{1 j k 1} \\
& \forall j=1, \ldots, N \\
& e_{j \ell} \geq t_{1 j} \cdot \sum_{k=1}^{K} x_{1 j k \ell}+\sum_{i=1}^{N} \sum_{k=1}^{K}\left(e_{i, \ell-1}+t_{i 0}+s_{i}\right) x_{i 0 k, \ell-1} \\
& \forall j=1, \ldots, N, \forall \ell=2, \ldots, L \\
& y_{i k \ell}, x_{i j k \ell} \in\{0,1\} \text {, } \\
& \forall i, j, \ell, k
\end{aligned}
$$

The objective (3) is to maximize the satisfaction and the objective (4) is to minimize the total travel time. Constraints (5) and (6) are flow conservation equations enforcing the route continuity. Constraints (7) show that in each period from node $i$ to node $j$ one kind of vehicle is used. Constraints (8) ensure that in each period if a vehicle $k$ enters into a node $j$, the same vehicle must leave the node. Constraints (9) and (10) guarantee that the total demand satisfied in each period be equal to the customer's demand. Constraints (9) ensure that only one vehicle is used to go from $i$ to $j$ in each period. Constraints (12), (18), (19), (20), (21) are the time window constraints ensuring that the vehicle be active at the particular time interval determined by each customer. Constraints (13) guarantee that the vehicle capacities are not exceeded. Constraints (14) eliminate the formation of sub-tours not containing the depot. Constraints (15) guarantee that if a customer is visited, then part of the demand should be satisfied. Constraints (16) show that the demand satisfied in each period must not exceed than the total demand minus the demands satisfied previously. Constraints (17) show that each vehicle is used merely for one trip. Constraints (22) give the binary restrictions. 


\subsection{Linearization of the model}

The nonlinear constraints (18) and (19) are linearized as follows:

$$
\begin{array}{ll}
p_{i j k l} \leq M x_{i j k l}, & \forall i, j=1, \ldots, N, j \neq i, \forall \ell, k \\
p_{i j k l} \geq t_{i j}-M\left(1-x_{i j k l}\right), & \forall i, j=1, \ldots, N, j \neq i, \forall \ell, k \\
p_{i j k l} \leq t_{i j}, & \forall i, j=1, \ldots, N, j \neq i, \forall \ell, k \\
p_{i j k l} \geq 0, & \forall i, j=1, \ldots, N, j \neq i, \forall \ell, k \\
e_{i \ell}-e_{j \ell} \leq M\left(1-\sum_{k=1}^{K} x_{i j k \ell}\right)-\sum_{k=1}^{K}\left(p_{i j k l}+s_{i} x_{i j k \ell}\right) & \forall i, j=1, \ldots, N, j \neq i, \forall \ell \\
e_{i \ell}-e_{j \ell} \geq-M\left(1-\sum_{k=1}^{K} x_{i j k \ell}\right)-\sum_{k=1}^{K}\left(p_{i j k l}+s_{i} x_{i j k \ell}\right) & \forall i, j=1, \ldots, N, j \neq i, \forall \ell
\end{array}
$$

\section{Computational Results}

Several examples were generated and used to examine the performance of the proposed model solved by the LINGO using the $\varepsilon$-constraint method. The initial data and the results obtained from solving these samples are presented in tables 1 and 2 . Figures 2 and 3 respectively show the Pareto points and the designed path.

Table 1. Initial data.

\begin{tabular}{|c|c|c|c|c|c|c|}
\hline Number of customers & Number of vehicles & Capacity & Demands (in order) & $\begin{array}{c}\text { Time windows } \\
\text { (in order) }\end{array}$ & $\begin{array}{c}\text { Service time } \\
\text { (in order) }\end{array}$ & Customer weights \\
\hline \multirow{3}{*}{4} & & & 0 & $(70-300)$ & 5 & 0.2273 \\
& 2 & 50 & 6 & $(30-350)$ & 3 & 0.2705 \\
& & & 200 & $(300-600)$ & 8 & 0.2077 \\
\hline
\end{tabular}

Table 2. Results (Pareto points).

\begin{tabular}{|c|c|}
\hline Objective 1 (satisfaction) & Objective 2 (time) \\
\hline 75.9469 & 329 \\
75.9437 & 320 \\
75.8567 & 315 \\
75.7924 & 300 \\
75.6742 & 294 \\
\hline
\end{tabular}

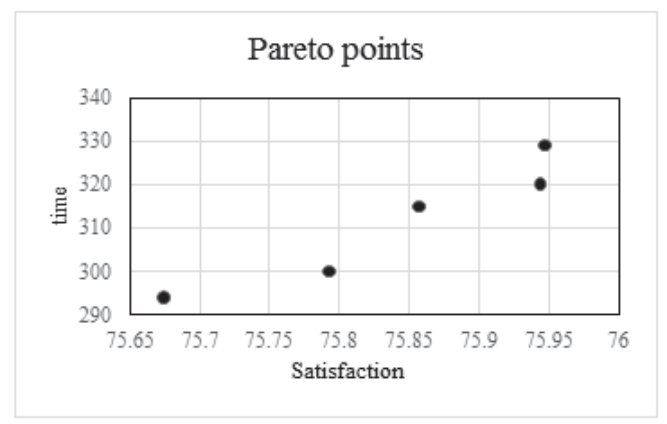

Figure 2. Pareto points 

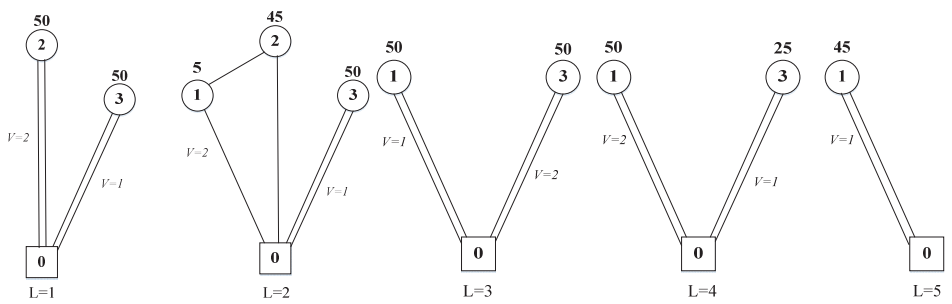

Figure 3-a. Designed path with satisfaction function
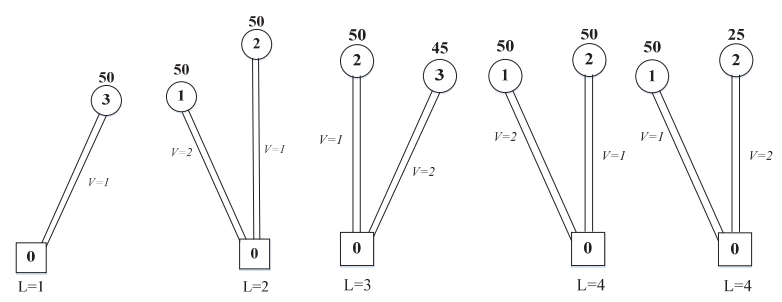

Figure 3-b. Designed path with time function

\section{Conclusion}

A bi-objective mixed-integer nonlinear programming model was presented in the context of split delivery vehicle routing problem with time window. To be practical, we considered resource limitations and allowed having more than one visit to satisfy the customer needs. Our aim was to maximize customer satisfaction and minimize travel time. This kind of a problem arises in distribution of essential commodities in conditions of scarcity. After linearization, the validation of the mathematical model was examined using the LINGO. For future studies, meta-heuristic approaches for large instances and multiple depots can be considered.

\section{Acknowledgements}

The first three authors thank Mazandaran University of Science and Technology and the last author thanks Sharif University of Technology for supporting this work.

\section{References}

[1]. Belhaiza, S., Hansen, P., Laporte, G., 2013. A hybrid variable neighborhood Tabu search heuristic for the vehicle routing problem with multiple time windows, Comput. Oper. Res. (in press), http://dx.doi.org/10.1016/j.cor.2013.08.010.

[2]. Bräysy, O., Gendreau, M. (2005a). Vehicle Routing Problem with Time Windows, Part I: Route Construction and Local Search Algorithms. Transportation Science, 39(1), 104-118 http://doi.org/10.1287/trsc.1030.0056

[3]. Bräysy, O., Gendreau, M. (2005b). Vehicle Routing Problem with Time Windows, Partll: Metaheuristics. Transportation Science, 39(1), 119-139 http://doi.org/10.1287/trsc.1030.0057

[4]. Koskosidis, Y.A., Powell, W.B., Solomon, M.M. (1992). An optimization-based heuristic for vehicle routing and scheduling with soft time window constraints, Transportation Sci. 26, 69-85.

[5]. Potvin, J.Y, Kervahut, T., Garcia, B.L., Rousseau, J.M. (1996). The vehicle routing problem with time windows, Part 1: tabu search, INFORMS J. Comput, 8 158-164.

[6]. Savelsbergh, M.W.P. (1992). The vehicle routing problem with time windows: minimizing route duration, ORSA J. Comput. 4, 146154.

[7]. Taillard, E., Badeau, P., Gendreau, M., Guertin, F., Potvin, J.Y. (1997). A tabu search heuristic for the vehicle routing problem with soft time windows, Transportation Sci. 31, 17186.

[8]. Martínez-Salazar, I., Angel-Bello, F., Alvarez, A. (2014). A customer-centric routing problem with multiple trips of a single vehicle, Journal of the Operational Research Society, 66(8), 1312-1323. 
[9]. Chen, P., Dong, X., Niu, Y. (2012). An iterated local search algorithm for the cumulative capacitated vehicle routing problem, in Tan, $\mathrm{H}_{\text {., }}($ Ed.), Technology for education and learning. Advances in intelligent and soft computing (pp. 575-581), Berlin/Heidelberg: Springer.

[10]. Moshref-Javadi, M., Lee Seokcheon, (2016). The customer-centric, multi-commodity vehicle routing problem with split delivery, Expert Systems with Applications, 56(1), 335-348

[11]. Dror, M., Trudeau, P. (1989). Savings by split delivery routing. Transportation Science, 23, 141-145

[12]. Dror, M., Trudeau, P. (1990). Split delivery routing, Naval Research Logistics, 37, 383-402.

[13]. Dror, M., Laporte, G., Trudeau, P. (1994). Vehicle routing with split deliveries, DiscreteApplied Mathematics, 50(3), $239-254$.

[14]. Frizzell, P. and J. Giffin (1995). The split delivery vehicle scheduling problem with time windows and grid network distances, Computers and Operations Research, 22(6), 655-667.

[15]. Archetti, C., Mansini, R., Speranza, M. G. (2001). The split delivery vehicle routing problem with small capacity, to appear in Transportation Science.

[16]. Archetti, C., Mansini,R., Speranza, M. G. (2005). Complexity and reducibility of the skip delivery problem, Transportation Science, 39(2), 182-187.

[17]. Archetti, C., Savelsbergh, M. W. P., Speranza, M. G. (2006). Worst-case analysis for split delivery vehicle routing problems, Transportation Science, 40(2), 226-234.

[18]. Archetti, C., Savelsbergh, M. W. P., Speranza, M. G. (2008). To split or not to split: That isthe question, Transportation Research, Part E 44(1), 114-123.

[19]. Belenguer, J., Martinez, M., Mota, E. (2000). A lower bound for the split delivery vehicle routing problem, Operations Research, 48(5), 801-810.

[20]. Jin, M., Liu, K., Eksioglu, B. (2008). A column generation approach for the split delivery vehicle routing problem, Oper. Res, Lett, 36(2), 265-270.

[21]. Archetti, C., Bianchessi, N., Speranza, M.G. (2014). Branch-and-cut algorithms for the split delivery vehicle routing problem. Eur. J. Oper. Res, 238(3), 685-698.

[22]. Silva, M.M., Subramanian, A., Ochi, L.S. (2015). An iterated local search heuristic for the split delivery vehicle routing problem, Comput. Oper. Res. 53(0), 234-249.

[23]. Han,A.F-H., Chu, Yu-Ching.(2016). A multi-start heuristic approach for the split-delivery vehicle routing problem with minimum delivery amounts, Transportation Research, Part E, 88, 11-31. 